\title{
Clean File
}

National Cancer Institute

\section{Source}

National Cancer Institute. Clean File. NCI Thesaurus. Code C142420.

A single file in which all errors have been detected and resolved. 